\title{
Enhancing the medical consultation with prior questions including ideas, concerns and expectations
}

\author{
Author: Mark Rickenbach
}

\begin{abstract}
Aims
Prior questions have the potential to make a consultation more focused, efficient and effective. This study aimed to design and assess the impact of a set of pre-consultation questions based on models of good consultations.
\end{abstract}

\section{Methods}

Pre-consultation questions were written based on existing principles of negotiation, education and being patient centred. These were piloted with doctors and patients. They were modified then applied to 100 consecutive consultations in two primary care settings over 2 weeks in September 2016.

\section{Results}

At consecutive routine primary care clinics question sheets were given to patients in the waiting area before being seen by a doctor. All patients who were asked agreed to participate. After initial review an additional question on duration of symptoms was added.

Out of 100 completed pre-consultation question sheets half were judged to have helped the consultation and none were reported to make it more difficult. The number of replies to each question varied from $100 \%$ to $69 \%$, with the exception of the question on duration of symptoms ( $42 \%$ replied). Doctors often had to specifically ask about symptom duration. Concerns were written down by $69 \%$ (69) of patients and 35\% (24) of these were not those expected by the doctor.

Application of pre-consultation questions was practical, all patients were positive and doctors reported consultations felt easier. Positive messages of support for the doctor were added to several sheets by patients. Patients reported it was helpful to prepare. For those with mild cognitive impairment, it helped to have a carer present.

\section{Conclusion}

Pre-consultation questions were a useful tool to help improve the focus and content of consultations. This format of questions has not been applied before in a combination that explains the limitations of the NHS, sets the agenda, and covers the ideas, concerns and expectations of patients. These prior questions are applicable to all forms of NHS consultations in healthcare, but most relevant to patients over 50 years of age attending a general medical clinic with multiple medical problems.

This was an observational, qualitative study and further research with a control group is required to determine if there is an overall time saving. As a result of this study patients are now offered online access to the question sheet and can access a copy by the appointment check-in terminal.

\section{Conflict of interest statement}

The author was also one of the participating doctors so there is a risk of researcher bias. The topic was a poster given a highly commended award at the Royal College of General Practitioners' conference at Harrogate October 2016. There was no financial support or sponsorship for this study. This abstract was submitted and accepted in 2017 for the RCP conference but the author was unable to present due to hip surgery. 